\title{
Descripción de pulsos de ecolocalización de Phyllostomus hastatus (Pallas, 1767) en un bosque húmedo tropical de San Francisco (Cundinamarca, Colombia)
}

\author{
Pulse description echolocation Phyllostomus hastatus \\ (Pallas, 1767) in a tropical rain forests of San Francisco \\ (Cundinamarca, Colombia)
}

\author{
Catalina Pinilla ${ }^{1}$, Abelardo Rodríguez-Bolaños ${ }^{2}$, Sergio Vogtschmidt ${ }^{3}$ \\ Resumen
}

\begin{abstract}
La interpretación de los sonidos ultrasónicos que emiten los murciélagos durante la fase de búsqueda puede proveer información sobre su identidad taxonómica y nos permite entender de una mejor manera cómo las especies hacen uso del hábitat, permitiendo su monitoreo. Objetivo: En este estudio, se describen ocho parámetros espectrales a partir del análisis de diez pulsos de ecolocación en fase de búsqueda emitidos por el murciélago lanceolado mayor Phyllostomus hastatus (Chiroptera: Phyllostomidae). Metodología: Los registros se obtienen mediante línea de vuelo y en espacio cerrado dentro de caseta de grabación. Se obtuvieron sonogramas visualizados a partir del software Sonobat 2.2 de dos indiviudos machos. Resultados: Se obtiene un consenso, entre todos los valores registrados, con la media y la desviación estándar entre los parámetros espectrales reportados por los dos individuos analizados; luego, dichos valores se confrontan, bajo algunas limitaciones, con lo reportado para P. hastatus por Baker (1977) y Santos (2003). Conclusión: Los sonogramas, tanto en espacio abierto como en espacio cerrado, comprenden pulsos cuya frecuencia mínima (Fmin/Lof) abarca rangos entre los $30-35 \mathrm{kHz}$ y máxima entre los $45-53 \mathrm{kHz}$. Los armónicos principales reportados desde el espacio abierto comprenden un rango de $42-50 \mathrm{kHz}$ y $38-45 \mathrm{kHz}$ en espacio cerrado; estos hallasgoz son consecuentes con lo reportado por Baker (1977) y Santos (2003).
\end{abstract}

Palabras clave: Phyllostomus hastatus, Registro acústico ultrasonido.

\begin{abstract}
Interpretation of ultrasonic sounds emitted by bats during the search phase can provide information on their taxonomic identity and allow us to understand better how the species make habitat use, allowing monitoring. Objective: Eight spectral parameters are described from analysis of ten phase echolocation pulses emitted by the search lanceolate greater bat Phyllostomus hastatus (Chiroptera: Phyllostomidae) in this study. Methodology: Records are obtained by flight line and enclosed space inside recording booth. They displayed sonograms were obtained from two software SonoBat 2.2 for two males. Results: Obtained consensus between all registered, with the average values and the standard deviation between the spectral parameters reported by the two individuals tested; subsequently, these values are compared, under some constraints, so for P. hastatus reported by Baker (1977) and Santos (2003). Conclusions: The sonograms in both open and closed space space, comprising pulses whose minimum frequency ( $\mathrm{Fmin} / \mathrm{Lof}$ ) covers ranges between $30-35 \mathrm{kHz}$ and $45-53 \mathrm{kHz}$ maximum between. The main harmonic reported from the open space comprise a range of $42-50 \mathrm{kHz}$ and $38-45 \mathrm{kHz}$ in enclosed space; these findings are consistent with those reported by Baker (1977) and Santos (2003).
\end{abstract}

Keywords: Phyllostomus hastatus, Ultrasound acoustic record.

1. Facultad de Ciencias y Educación, Universidad Distrital Francisco José de Caldas, Bogotá, Colombia. Grupo de Investigación Biodiversidad de Alta Montaña. e-mail: pcpinillac@correo.udistrital.edu.co

2. Facultad de Ciencias y Educación, Museo de Historia Natural (MHNUD), Universidad Distrital Francisco José de Caldas, Bogotá, Colombia. e-mail: abelardorodriguezb@gmail.com

3. Museo de Historia Natural Noel Kempff Mercado. Área de Evolución y Paleontología, Santa Cruz, Bolivia. e-mail: s.vogtschmidt@gmail.com Fecha recepción: Noviembre 25, 2013 Fecha aprobación: Diciembre 13, $2013 \quad$ Editor asociado: Mantilla H. 


\section{Introducción}

La emisión de ultrasonidos constituye un atributo biológico que permite a varias especies de murciélagos llevar a cabo gran cantidad de las tareas. Dichas emisiones tienen un rol determinante en la interpretación de su entorno natural, permitiendo el desplazamiento, la orientación, así como la obtención de alimento. El tipo de emisiones más frecuente entre murciélagos, y que describen su actividad principal, son las de fase de búsqueda que constituyen cerca del 90\% de la actividad emisora de los murciélagos (Mora 2002); estas emisiones presentan pocas variaciones por lo que son las empleadas para la identificación taxonómica. Es así como la interpretación de parámetros que describen dichos ultrasonidos arroja información significativa sobre la identidad o huella acústica de las especies y permite estimar la actividad de los individuos en un momento determinado (Rivera-Parra 2013).

Phyllostomus hastatus es la segunda especie de mayor tamaño en el neotrópico (antebrazo 82.0-83.9 mm N= 80) (Eisenberg 1989; Santos 2003; Aguirre 2007). De las tres especies descritas de Phyllostomus esta es la de mayor tamaño, la longitud del cuerpo supera los $105 \mathrm{~mm}$, el largo del antebrazo es mayor a $68 \mathrm{~mm}$, su hoja nasal es simple y bien desarrollada, el centro del labio con verrugas, orejas triangulares puntiagudas y bien separadas. Los machos presentan un saco glandular en la garganta el cual es rudimentario en hembras, el color de su pelaje es uniforme ventral y dorsalmente, este puede variar de negro a café rojizo, la membrana alar se halla adherida al metatarso, el calcáneo se encuentra ampliamente desarrollado y supera el tamaño del pie, su cola es corta y su ápice sobresale por encima del uropatagio (Baker 1977).

En Colombia varios autores reportan esta especie en la mayoría de los pisos térmicos y hábitats (Mantilla-Meluk et al. 2009), incluyendo zonas húmedas hasta semiáridas en localidades de Cundinamarca, Bogotá, San Javier y Villeta (Thomas 1924, Valdivieso 1964, Valdivieso 1964).

La dieta de $P$. hastatus es principalmente omnívora, abarcando pequeños vertebrados como ratones, pájaros, otros murciélagos, invertebrados, frutos, polen y partes florales (Aguirre 2007). Muñoz (2001), reporta picos de mayor actividad durante las primeras horas de la noche entre las 20:00 y 22:00 h.

Ecolocación en P. hastatus. Para la familia Phyllostomidae, Kalko (2002) reconoce que el mecanismo de ecolocación presenta estructuras estereotipadas, bajas intensidades y elevadas frecuencias, lo cual dificulta el análisis e identificación de especies. Según Santos (2003), los pulsos en fase de búsqueda de $P$. hastatus presentan frecuencias moduladas, de corta duración y baja intensidad, con frecuencia característica cerca a los $40 \mathrm{kHz}$, sus emisiones cubren un amplio ancho de banda, es decir abarcan una amplia gama de frecuencias las cuales pueden solaparse, los sonogramas pertenecientes a $P$. hastatus, muestran picos entre $30-35 \mathrm{kHz}$ y de $50-55 \mathrm{kHz}$, con armónicos que abarcan una gama de frecuencias entre $42-50 \mathrm{kHz}$.

En el presente estudio analizamos información asociada con el primer registro de $P$. hastatus en un bosque húmedo montano bajo ubicado en el municipio de San Francisco, Cundinamarca, Colombia.

\section{Metodología}

Sitio de muestreo. El municipio de San Francisco pertenece al noroccidente del departamento de Cundinamarca, limita por el norte con Supatá y La Vega, por el oriente con Subachoque y por el sur con Facatativá. La zona de estudio se encuentra en la vereda San Miguel, ubicada en la parte norte del municipio sobre la formación de bosque húmedo montano, típicamente evidenciando zonas de montaña fértiles y pedregosas, relieve quebrado donde predomina el suelo con vegetación permanente y acuíferos representativos (Figura 1). La precipitación media anual varía entre los 900 y $1800 \mathrm{~mm}$, las lluvias por lo general son irregulares pero logran mantener la humedad en el terreno. La temperatura es moderada y propia de clima templado, oscila entre 12 y $20^{\circ} \mathrm{C}$. El municipio incluye un rango de elevación entre los 2300 y 2600 m.

Capturas. Durante la primera sesión de muestreo, que se llevó a cabo en septiembre de 2013, se instalaron dos redes de niebla de $12 \mathrm{~m}$ y $6 \mathrm{~m}$, en esta última es capturado un macho (Figura 2), con testículos desarrollados de $P$. hastatus sobre las 8:40 pm, que reportó las siguientes medidas: antebrazo $(\mathrm{AB})=88,26$ $\mathrm{mm}$; longitud de la oreja $(\mathrm{LO})=31 \mathrm{~mm}$; longitud de la hoja nasal $(\mathrm{LHN})=16 \mathrm{~mm}$. Un segundo especímen 

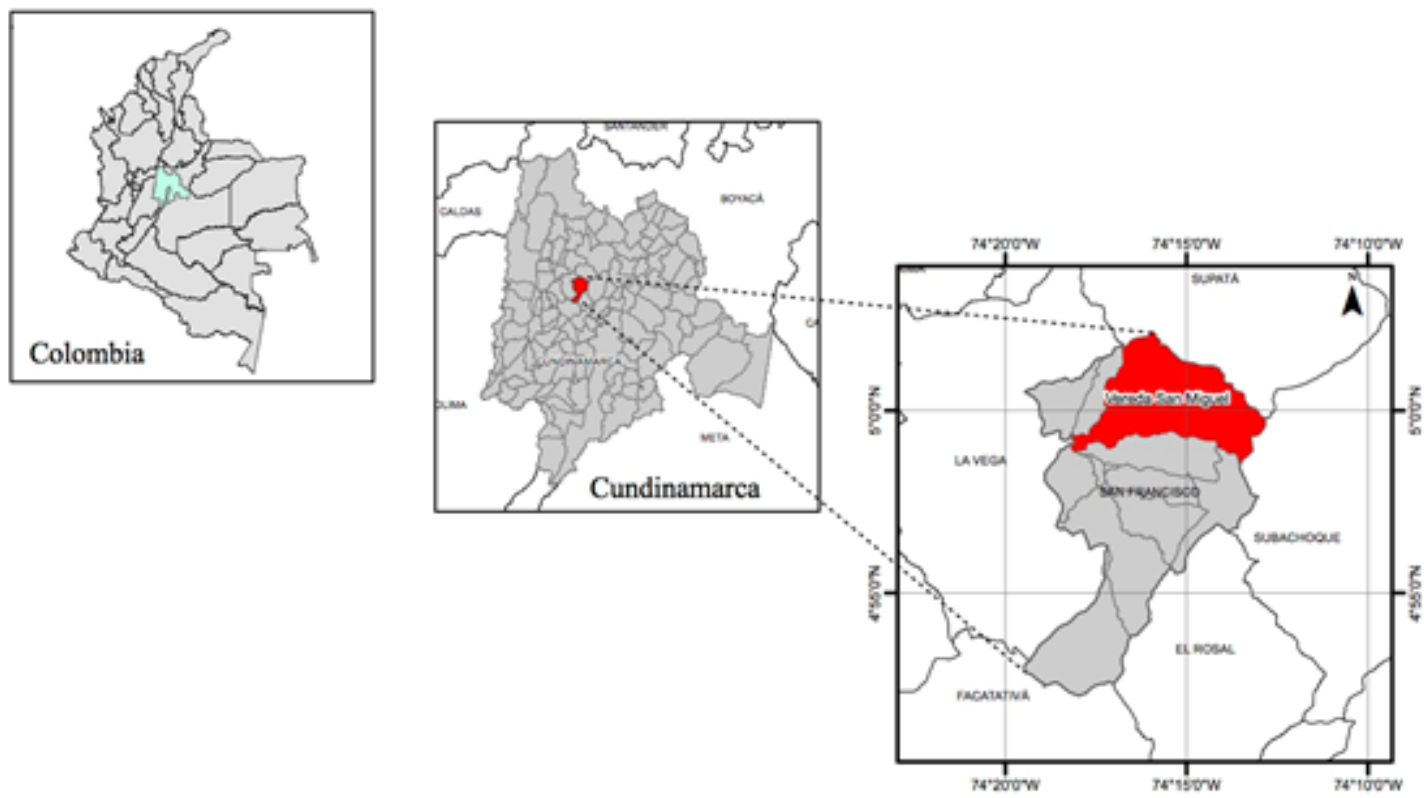

Figura 1. Mapa de la zona de estudio.

Recuperado el 24 de noviembre de 2013 (http://sanfrancisco-cundinamarca.gov.co/mapas_municipio.shtml) y XI Encuentro Nacional de Capacitación en Ganadería, Agroecológica y Pastoreo Racional (VOISIN) (http://www.culturaempresarialganadera.org/forum/topics/experiencia-con-prv-en-sanfrancisco-de-sales-cundinamarca)

macho, testiculado, es capturado en noviembre de 2013 en una red de $6 \mathrm{~m}$, sobre las 9:10 pm con las siguientes medidas: antebrazo $(\mathrm{AB})=87,5 \mathrm{~mm}$; longitud de la oreja $(\mathrm{LO})=32 \mathrm{~mm}$; longitud de la hoja nasal $(\mathrm{LHN})=16,5 \mathrm{~mm}$.

Ecolocación. Para registrar las señales ultrasónicas en fase de búsqueda se empleó el método de línea de vuelo que consiste en ubicar dos varas que pueden ser de metal o de madera en un espacio abierto a una distancia aproximada de $5 \mathrm{~m}$ una de la otra (Figura 3). Entre las dos varas fue extendida una hebra de igual longitud donde fue insertada una argolla metálica, la cual se encontraba a su vez atada a una hebra de nylon elástico de 1,5 m cuyo extremo será atado a manera de lazo en el cuello del murciélago con el objetivo que este pueda desplazarse sobre la línea de vuelo procurando que quede lo suficientemente ajustado para no lastimar al individuo y evitar que pudiese escapar mientras se realiza el registro acústico (Rivera-Parra 2013).

Un segundo experimento fue realizado registrando las vocalizaciones de un segundo espécimen en una caseta de vuelo de $4 \times 4 \mathrm{~m}$, construida con polisombra. Para facilitar el vuelo controlado en el espacio cerrado se dispone de un palo de madera, a partir del cual se amarra uno de los extremos de una hebra

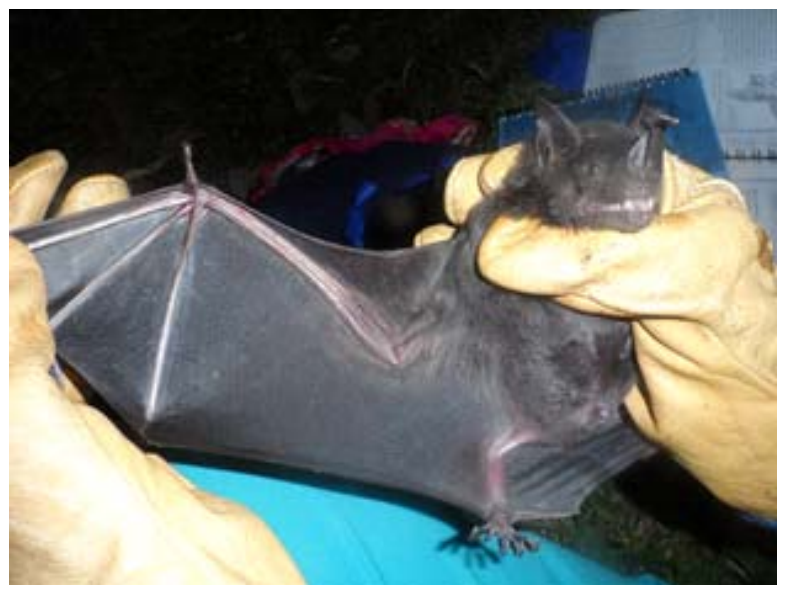

Figura 2. Especímen de Phyllostomus hastatus, macho adulto capturado y liberado en septiembre de 2013.

elástica semigruesa de una longitud aproximada de $2.5 \mathrm{~m}$. El otro extremo de la hebra, se ajustó con precaución al cuello del murciélago (Figura 4). Al igual que el método de línea de vuelo se procuró realizar el lazo lo suficiente justo para que el individuo no escape y que de igual manera no pudiese afectarle.

Reporte acústico, selección de pulsos y análisis. Para registrar los ultrasonidos fue empleada una grabadora Autonoma Song Meter SM2BAT (Winfred y Frick 2013), Wild Life (Ossa 2010). La visualiza- 


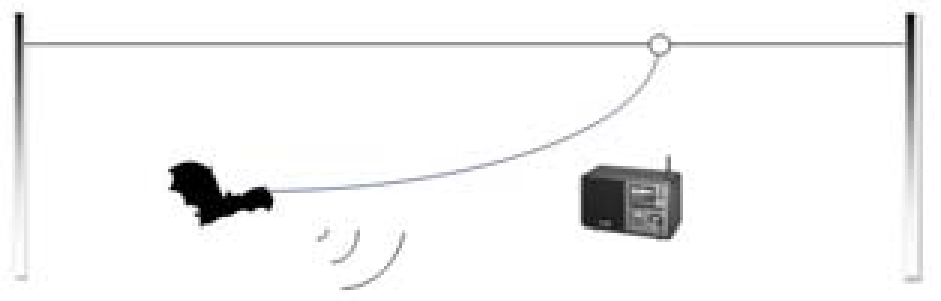

Figura 3. Diagrama de registro en línea de vuelo modificado de Rivera-Parra 2013.

ción, selección y caracterización de las emisiones ultrasónicas registradas se llevó a cabo con una tasa de muestreo de $192 \mathrm{kHz}$ mediante el software Sonobat 2.2 (Software for Bat call Analysis 2004).

\section{Resultados y discusión}

Sonogramas. En los sonogramas obtenidos se visualizó: 1) la estructura y forma de la frecuencia modulada (FM); frecuencia constante (FC); y cuasiconstante (QCF), que vincula los dos anteriores (Figura5).

Durante el manejo cuantitativo obtuvimos valores espectrales (Rivera-Parra 2013) de: 1) frecuencia más alta (Hif), 2) frecuencia más baja (Lof), 3) frecuencia máxima (Fmax), 4) frecuencia del punto de inflexión del pulso (Knee), 5) porción de la emisión con mayor intensidad $(\mathrm{ldg}), 6)$ frecuencia característica $(\mathrm{Fc}), 7)$ duración del pulso $(\mathrm{ms}), 8)$ primer y segundo armónico (h1) (Figura 6).

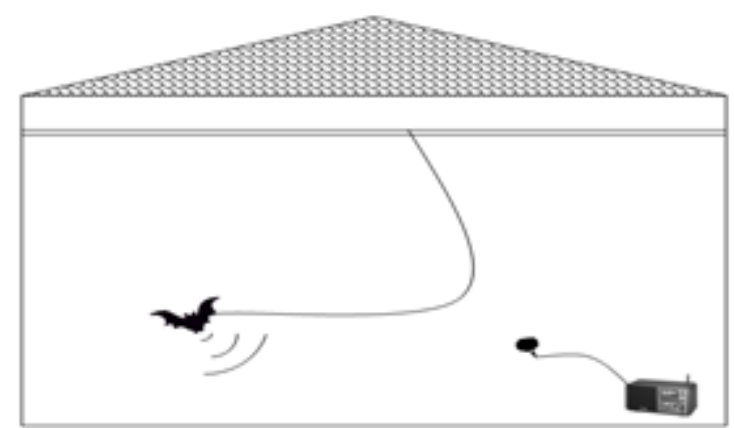

Figura 4. Diagrama de registro en la caseta de grabación.

Se describen parámetros para el método de registro en espacio abierto con la línea de vuelo (Tabla 1), como para el método de espacio cerrado en caseta de grabación (Tabla 2). Para el análisis se ha tenido en cuenta valores espectrales de cada pulso, así como descriptivos como la media y la desviación estándar para cada parámetro espectral. Obtuvimos un consenso y estimamos la heterogeneidad de las vocalizaciones con el promedio y desviación estándar entre

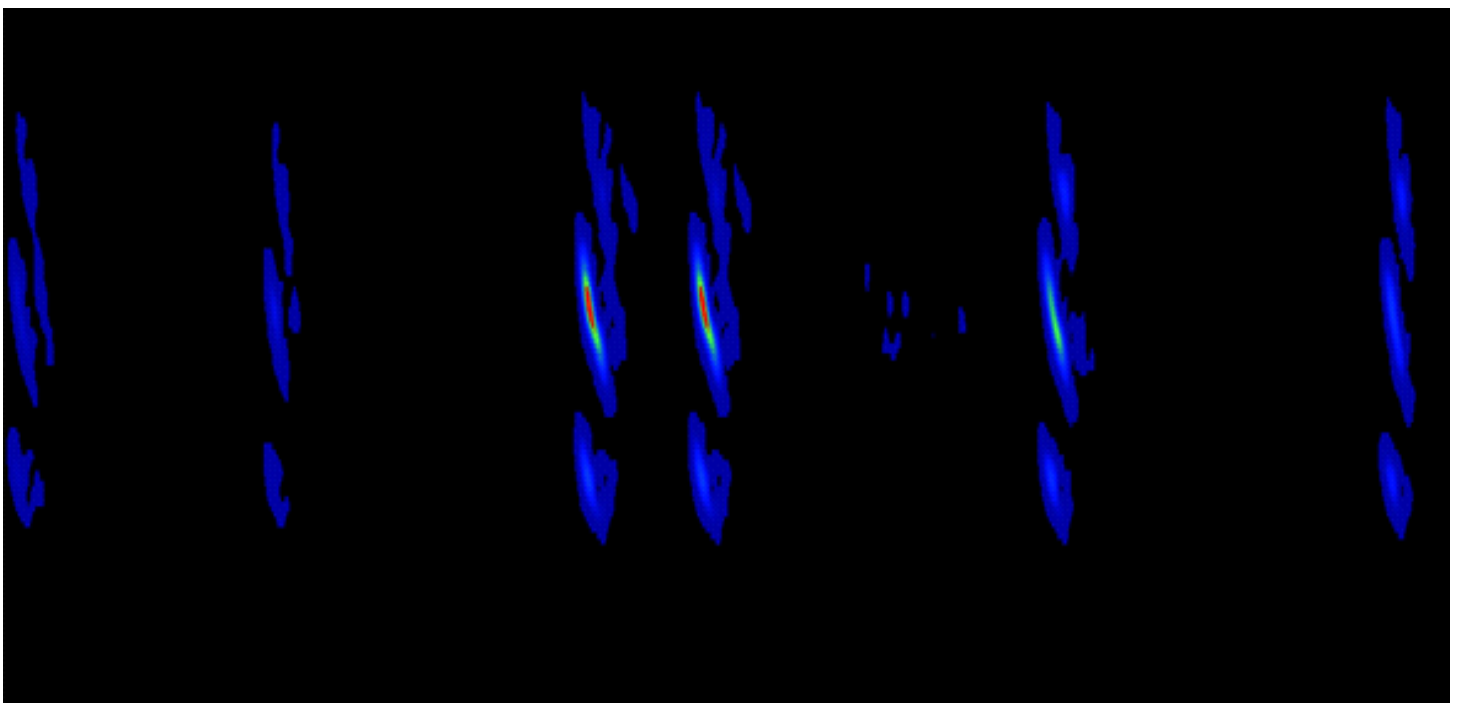

Figure 5. Secuencia de seis pulsos consecutivos de $P$. hastatus, vista a partir del sonobat 2.2 Software for Bat call Analysis (2004). 


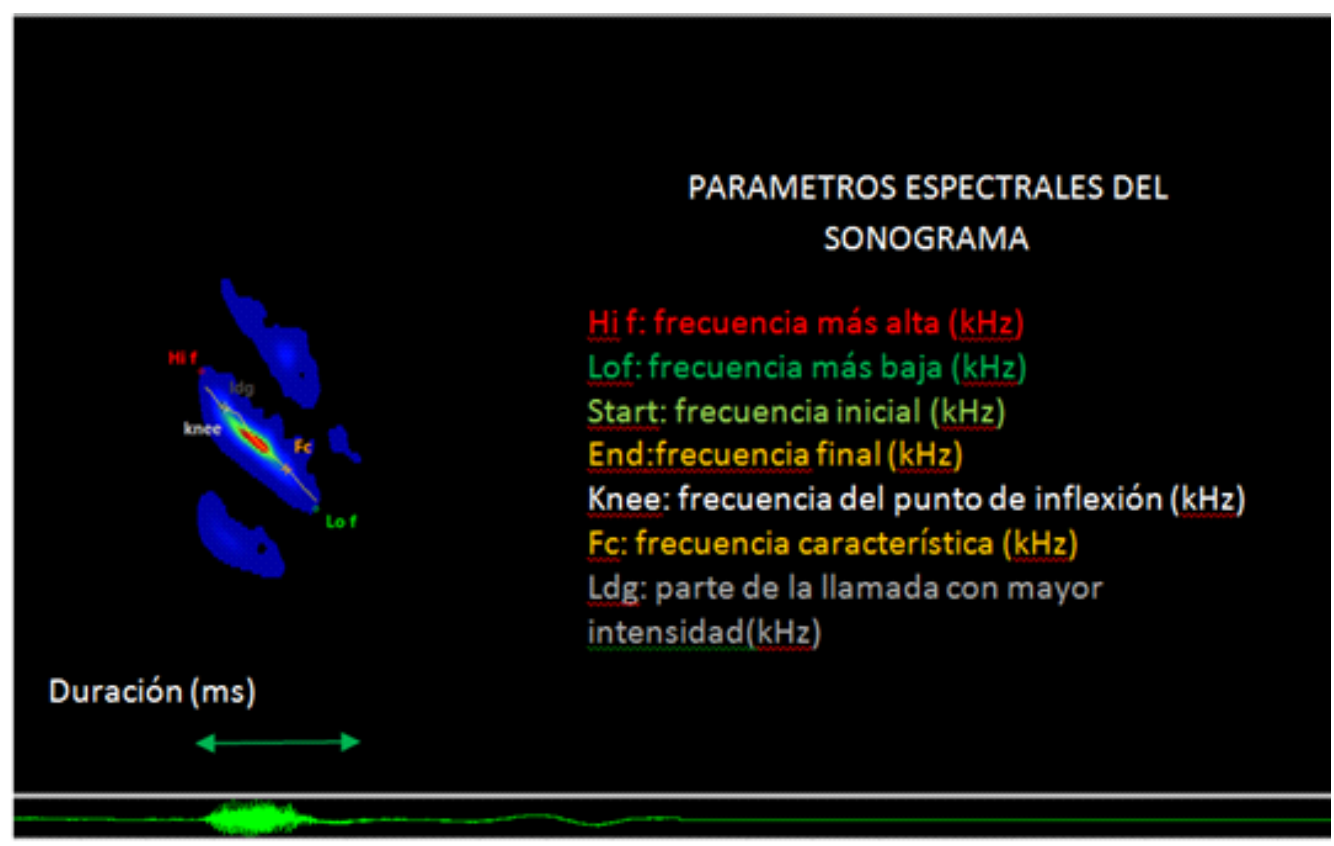

Figura 6. Parámetros espectrales de uno pulso sometido a análisis.

los diez pulsos en cada unos de los métodos de registro (Tablas 3 y 4). Posteriormente, se realiza una comparación con limitaciones, teniendo en cuenta datos acústicos de la especie reportados por Baker (1977) y Santos (2003).

Baker (1977) reporta cuatro parámetros de los seis que fueron sometidos a comparación en este estudio mientras que Santos (2003) reporta cinco. Los valores correspondientes a los parámetros espectrales que fueron contrastados son: frecuencia máxima Fmax o (Hif), frecuencia mínima Fmin o Lof, duración del pulso, armónico, frecuencia característica o Fc y cualitativamente se tuvo en cuenta la estructura del pulso. Los autores que reportan estos datos no describen una metodología de registro en particular, de esta manera la comparación se realiza de forma teórica y teniendo en cuenta únicamente los parámetros presentados por los autores.

Cualitativamente, se corroboró que la estructura de las emisiones de P. hastatus son de frecuencia modulada tanto en espacio abierto como en espacio cerrado. Lo que coincide con lo reportado por Baker (1977) y Santos (2003), y abarcan distintos valores de frecuencias en un tiempo muy corto.

Santos (2003) y Baker (1977), describen los

Tabla 1. Valores espectrales asociados con cada pulso, teniendo en cuenta registros de línea de vuelo.

\begin{tabular}{|c|c|c|c|c|c|c|c|c|}
\hline & \multirow[t]{2}{*}{$\mathrm{HiF}(\mathrm{kHz})$} & \multirow[t]{2}{*}{ LoF (kHz) } & \multirow[t]{2}{*}{ F@max } & \multirow[t]{2}{*}{ Fc (kHz) } & \multirow[t]{2}{*}{ Knee (kHz) } & \multirow[t]{2}{*}{ FreqLdg (kHz) } & \multicolumn{2}{|c|}{ Duration } \\
\hline & & & & & & & (ms) & h1(kHz) \\
\hline 1 & 48,05874 & 36,1043 & 43,9649 & 36,39142 & 37,870746 & 36,902199 & 1,5028 & 44 \\
\hline 2 & 47,31416 & 36,9162 & 44,3063 & 37,10077 & 41,45772 & 37,849432 & 1,110063 & 44,3 \\
\hline 3 & 49,42818 & 36,1808 & 44,8007 & 36,73286 & 44,80069 & 37,506805 & 1,681389 & 44,8 \\
\hline 4 & 48,86785 & 35,9386 & 45,5859 & 36,11936 & 39,894101 & 39,894101 & 1,109754 & 45,6 \\
\hline 5 & 50,54462 & 36,5992 & 42,4083 & 37,47653 & 47,017427 & 37,796002 & 1,225936 & 42,4 \\
\hline 6 & 47,39044 & 36,1907 & 44,8064 & 36,75831 & 43,060938 & 37,056639 & 1,472738 & 44,8 \\
\hline 7 & 50,0209 & 36,0133 & 45,1793 & 36,77384 & 39,612245 & 47,513659 & 1,666863 & 45,2 \\
\hline 8 & 47,86402 & 36,7178 & 43,2795 & 38,14338 & 41,514318 & 43,543655 & 0,99228 & 43,3 \\
\hline 9 & 50,31732 & 36,1067 & 44,267 & 40,12974 & 47,660725 & 47,892057 & 1,652609 & 43,3 \\
\hline 10 & 49,48239 & 35,8619 & 42,6364 & 36,75251 & 43,721957 & 37,086069 & 1,694592 & 42,6 \\
\hline
\end{tabular}


Tabla 2. Valores espectrales asociados con cada pulso, teniendo en cuenta registros en la caseta de grabación.

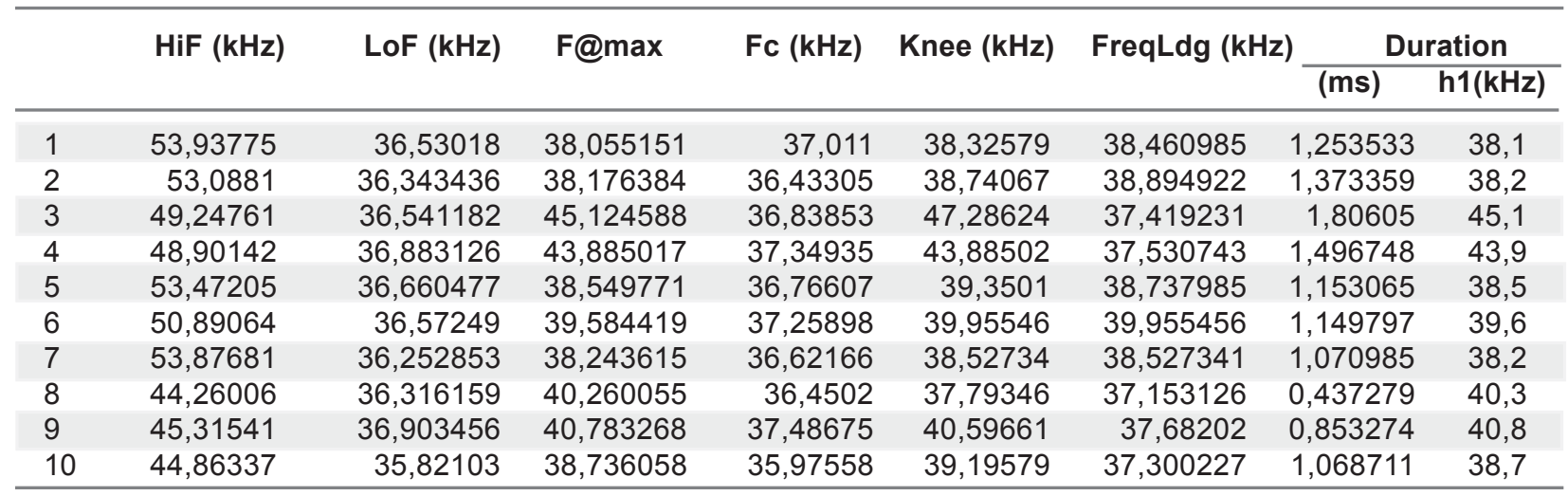

Tabla 3. Valores de cada parámetro espectral (media $\pm \mathrm{SD}$ ), asociados con los reportes en línea de vuelo.

\begin{tabular}{lc}
\hline Hi $f(k H z)$ & $48,9 \pm 1,20$ \\
Lof $(\mathrm{kHz})$ & $36,26 \pm 0,35$ \\
F@ $\max (\mathrm{kHz})$ & $44,12 \pm 1,06$ \\
$\mathrm{Fc}(\mathrm{kHz})$ & $37,23 \pm 1,11$ \\
Knee $(\mathrm{kHz})$ & $42,66 \pm 3,20$ \\
FreqLedldg $(\mathrm{kHz})$ & $40,30 \pm 4,38$ \\
Duracion $(\mathrm{ms})$ & $1,41 \pm 0,27$ \\
Armonico h1 $(\mathrm{kHz})$ & $44,03 \pm 1,09$ \\
\hline
\end{tabular}

pulsos de $P$. hastatus como señales de corta duración y frecuencia modulada. Respecto a sus parámetros acústicos y según Santos (2003), exhiben una frecuencia característica cercana a los $40 \mathrm{kHz}$, este índice es consistente con nuestros hallazgos mediante el método aplicado en espacio abierto, evidenciando un rango de (36-40 kHz). Al obtener sonogramas tanto en espacio abierto como en espacio cerrado, en este estudio se obtienen pulsos cuya frecuencia mínima (Fmin/Lof) comprende rangos entre los 30-35 kHz y su frecuencia máxima abarca una gama de frecuencias entre los 45-53 kHz. Los armónicos principales reportados desde el espacio abierto comprenden un rango de $42-50 \mathrm{kHz}$ y $38-45 \mathrm{kHz}$ en espacio cerrado.

En ambos casos los rangos arrojados por los armónicos, que son repeticiones de la frecuencia modulada a la cual se encuentra el máximo valor de energía, resultan ser coherentes con los propuestos por Santos (2003) que están en un marco de 42-50 kHz.

Según Baker (1977) los pulsos de P. hastatus presentan una duración entre $0,5-4,0 \mathrm{~ms}$, lo cual resulta
Tabla 4. Valores de cada parámetro espectral (media \pm SD), asociados con reportes en la caseta de grabación.

\begin{tabular}{lc}
\hline HiF $(\mathrm{kHz})$ & $49,78 \pm 3,88$ \\
LoF $(\mathrm{kHz})$ & $39,48 \pm 0,31$ \\
$\mathrm{~F} @$ max & $40,13 \pm 2,49$ \\
$\mathrm{Fc}(\mathrm{kHz})$ & $36,89 \pm 0,47$ \\
Knee $(\mathrm{Khz})$ & $40,37 \pm 2,97$ \\
FreqLdg $(\mathrm{kHz})$ & $38,166 \pm 0,89$ \\
Duration $(\mathrm{ms})$ & $1.16 \pm 0,36$ \\
h1 $(\mathrm{Khz})$ & $40,14 \pm 2,49$ \\
\hline
\end{tabular}

coherente al realizar reportes tanto en espacio abierto como cerrado, donde se obtienen valores entre los 0.99 y $1.6 \mathrm{~ms}$ y $0,4-1.8 \mathrm{~ms}$ respectivamente.

Los patrones acústicos encontrados coinciden con aspectos teóricos generales como los que explican que los barridos que comprenden varias frecuencias contribuyen a incrementar la habilidad de discriminación de los objetos que hacen parte del entorno de los individuos cuando están realizando actividades de exploración. Las frecuencias moduladas se emplean con frecuencia por individuos pertenecientes a la familia Phyllostomidae, por sus requerimientos de orientación las señales principales suelen estar acompañadas de armónicos (h) Hill (1986), que son repeticiones de la frecuencia característica $(\mathrm{Fc})$ emitida por el animal durante la fese de búsqueda y también son el resultado de la capacidad de vibración simultánea que presentan las estructuras vocales emisoras de los ultrasonidos complementando la frecuencia fundamental de la emisión (Airas 2003). 


\section{Conclusiones}

Al comparar nuestros hallazgos con los parámetros reportados en otras investigaciones y teniendo en cuenta las referencias de frecuencia máxima (Fmax/Hif) los datos obtenidos empleando el método de línea de vuelo en espacio abierto corresponden a $47-50 \mathrm{kHz}$ y son coherentes con los presentados por Baker (1977), con rango $42-50 \mathrm{kHz}$, mientras que los valores para frecuencia máxima obtenidos al realizar el registro en espacio cerrado son de $45-53 \mathrm{kHz}$ y caen en el rango de $50-55 \mathrm{kHz}$ que reporta Santos (2003).

\section{Literatura citada}

Airas M. 2003. Echolocation in bats. HUT. In: Proceedings of spatial sound perception and reproduction. The postgrad seminar course of HUT Acoustics Laboratory. p. 1-25.

Albuja LV. 1999. Murciélagos del Ecuador. Quito: Editorial Escuela Politécnica Nacional.

Aguirre LF. 2007. Historia natural, distribución y conservación de los murciélagos de Bolivia. Santa Cruz de la Sierra: Centro de Ecología y Difusión Fundación. Simón Patiño, SIRENA. 416 pp.
Baker RJ, Jones JKJr, Carter DC (eds.). 1977. Biology of bats of the New World family Phyllostomidae Par II. Publicación especial de la Museum, Texas Tech University. 364 pp.

Balmori MA. 1998. El estudio de los quirópteros a través de sus emisiones ultrasónicas. Métodos en mastozoología. Galemys 10 (1): 12-9.

Frick W. 2013. Acoustic monitoring of bats, considerations of options for long-term monitoring. Therya 4 (1): 69-78.

Hill JE. (1986). Bats a natural history. Texas: University of Texas, British Museum of Natural History.

Kalko E. 2002. Neotropical leaf-nosed bats (Phyllostomidae): «Whispering» bats as candidates for acoustic surveys? In: Brigham MK (ed). Bat echolocation research tools, tecniques and analysis. Austin: Bat Conservation International. p. 63-71.

Muñoz AJ. 2001. Los murciélagos de Colombia, sistematica, distribucion, descripción, historia natural y ecología. Medellín: Editorial Universidad de Antioquia.

Ossa GG. 2010. Métodos bioacústicos: una aproximación a la ecología de comunidades de murciélagos en las ecoregiones mediterránea y el bosque templado de Chile. Tesis de grado. Santiago: Facultad de Agronomía e Ingeniería Forestal, Pontificia Universidad Católica de Chile.

Rivera-Parra B. 2013. Primera biblioteca de llamadas de ecolocación de murciéalgos del Ecuador. Therya. 4 (1): 79-88.

Santos M, Aguirre LF, Vásquez L, Ortega J. 2003. Phyllostomus hastatus. Mammalian Species. 722: 1-6. 